\title{
An Ovarian Cancer Patient Presenting Heparin-Induced Thrombocytopenia After Use of Low Molecular Weight Heparin: A Case Report of Rare Condition
}

\author{
Yasin DURMUS'1, Yalin Ay KARYAL ${ }^{1}$, Cigdem KILIC ${ }^{1}$, Caner CAKIR ${ }^{1}$, Dilek YUKSEL ${ }^{1}$, Berna SEYHAN', \\ Gokhan BOYRAZ1, Gunsu KIMYON COMERT'1 Taner TURAN1
}

Ankara, Turkey

\begin{abstract}
Heparin-induced thrombocytopenia is a rare and life-threatening condition of exposure to heparin. A case of heparin-induced thrombocytopenia due to the low molecular weight heparin was presented. Pulmonary emboli and progressively decreased number of thrombocytes developed during the low molecular weight heparin treatment. For that reason, the heparin-induced thrombocytopenia was diagnosed. The heparin was ceased and fondaparinux treatment initiated. Platelet levels returned to normal limits within six days. The delaying in diagnosis of heparin-induced thrombocytopenia causes serious outcomes. The physician must be careful and keep in mind be developed of this clinical condition in patient under heparin treatment.
\end{abstract}

Keywords: Heparin-induced thrombocytopenia, Low molecular weight heparin, Ovarian cancer

Gynecol Obstet Reprod Med 2019;25(3):176-179

\section{Introduction}

Heparin-induced thrombocytopenia (HIT) is an immunemediated, life-threatening condition of exposure to heparin (unfractionated heparin; UFH or low molecular weight heparin; LMWH) that occurs in a small percentage of patients exposed, regardless of the dose or administration (1). HIT re-

${ }^{1}$ Health Sciences University Etlik Zubeyde Hanim Women's Health Education and Researching Hospital Gynecologic Oncology Clinic, Ankara, Turkey

Address of Correspondence: Yalin Ay Karyal

Health Sciences University, Etlik

Zubeyde Hanim Women's Health

Teaching and Research Hospital, Gynecologic Oncology Division, 06010 Etlik, Ankara Turkey yalinay.kryl@gmail.com

Submitted for Publication: $\quad 31.05 .2018$

Revised for: Publication $\quad 26.08 .2018$

Accepted for Publication: $\quad 15.10 .2018$

Author Contributions: $\quad Y D: 0000-0002-5404-0118$,

YAK: 0000-0002-5088-2334, CK: 0000-0002-4433-8068,

CC: 0000-0003-2559-9104, DY: 0000-0002-2366-8412,

BS: 0000-0000-0000-0000, GB: 0000-0001-6165-1497,

GKC: 0000-0003-0178-4196, TT: 0000-0001-8120-1143

\begin{tabular}{|c|c|}
\hline Quick Response Code: & Access this article online \\
\cline { 2 - 2 } & Website: www.gorm.com.tr \\
& e- mail: info@gorm.com.tr \\
\cline { 2 - 3 } & DOI:10.21613/GORM.2018.799 \\
\hline
\end{tabular}

How to cite this article: Durmus Y. Karyal YA. Kilic C. Cakir C. Yuksel D. Seyhan B. Boyraz G. Kimyon Comert G. Turan T. An Ovarian Cancer Patient Presenting Heparin - Induced Thrombocytopenia after use of Low-Molecular - Weight Heparin: A Case Report of Rare Condition. Gynecol Obstet Reprod Med 2019;25(3):176-179 sults from an autoantibody directed against endogenous platelet factor 4 (PF4) in complex with heparin. HIT antibodies remain in blood circulation for several weeks to several months (2). This immune reaction activates platelets and can increase the risk of catastrophic arterial and venous thrombosis. HIT is estimated to occur in $<0.1$ to $5.0 \%$ of patients receiving heparin depending on the clinical scenario (1-3). Unfractionated heparin and LMWH are commonly used agents for prophylaxis against venous thromboembolism after surgery and in patients admitted to the hospital (3). A potential advantage of LMWH from clinical and cost perspectives is a lower risk for HIT (4).

The most important risk factors for HIT are; duration of heparin therapy, type of heparin, indication for UFH/LMWH, and sex of the patient (5). Clinically, HIT may lead to asymptomatic or symptomatic thrombocytopenia or manifest as skin lesions at the heparin injection site or with acute systemic reactions like chills, fever, dyspnea, chest pain, bleeding (intracranial/retroperitoneal) or thrombosis like pulmonary embolism, myocardial infarction, cerebrovascular disease, necrosis of extremity.

The following steps should be followed when diagnosing HIT; evidence of longer than 5 days' heparin exposure, omission of other causes of thrombocytopenia, demonstration of HIT antibodies by functional or antigenic methods (ELISA, serotonin release assay, heparin-induced platelet aggregation test, flow cytology) and elevated platelet counts 4 to 14 days after heparin is discontinued should be observed.

This case report is written to indicate that HIT should al- 
ways be remembered when a patient's platelet counts decrease after 5-10 days of heparin treatment. Here is described the case of a patient with HIT syndrome due to LMWH use after an ovarian cancer surgery.

\section{Case Report}

A 74-year-old patient underwent surgery for advanced stage ovarian cancer. Intraoperative frozen section assessment of omental cake was reported as malign epithelial tumor and advanced stage ovarian cancer was confirmed. Patient had comorbidities like diabetes mellitus, hypertension and poor medical condition for a debulking surgery so we have decided to administer neoadjuvant chemotherapy. For that reason, due to the choice of senior surgeon, infracolic omentectomy, right salpingectomy, umbilical hernia repair was performed within the context of initial surgery. A weight and age-adjusted dose of LMWH (4000 anti-Xa IU $40 \mathrm{mg}$ enoxaparin sodium) was initiated for deep vein thrombosis prophylaxis during post-operative periods. Afterwards the surgery, chemotherapy treatment was planned according to pathology and patient was discharged with LMWH treatment; which as a protocol, was prescribed for a month after surgery. Her final histopathology result was high grade serous ovarian carcinoma and thus she received chemotherapy (paclitaxel $=175 \mathrm{mg} / \mathrm{m}^{2}$ and carboplatin $=$ area under curve $=6$; per three weeks). After 4 cycles of chemotherapy, she underwent an interval debulking surgery procedure, which was required and received the $5^{\text {th }}$ chemotherapy cycle on the 10th day of post-operative followup. Type 2 hysterectomy + left salpingo-oophorectomy + right oophorectomy + bilateral pelvic and paraaortic lymph node dissection + total omentectomy + appendectomy + splenectomy + tumoral tissue resection from diaphragm were performed within the context of interval surgical procedure. The following day of interval surgery, LMWH (4000 Anti-Xa IU/0.4 MI) was initiated for prophylaxis and the patient was discharged with the follow-up arrangements. On the $6^{\text {th }}$ day of outpatient follow-up after receiving the $5^{\text {th }}$ cycle of chemotherapy, she was admitted to hospital with complaints of nausea, vomiting, diarrhea, and fever. Due to clinical examination as fever, oral aphthous ulcer was revealed; she was hospitalized for inpatient treatment and consulted to the infectious disease department. The clinical status of the patient with normal hematologic parameters was attributed to chemotherapy toxicity and gastrointestinal toxicity. IV antibiotics and fluid retention were administered and so clinical improvement achieved.

She has received the $6^{\text {th }}$ cycle of chemotherapy and after 10 days of accomplished cycle, she underwent examination and laboratory results indicated that her platelet count declined to $9000 / \mathrm{mm}^{3}$ and the thrombocytopenia was revealed. Other parameters were in the normal range. Thrombocyte replacement (6 units) was performed considering the hematological toxicity. After the replacement, platelet count has in- creased to $112.000 / \mathrm{mm}^{3}$. But the platelet count decreased to $34.000 / \mathrm{mm}^{3}$ within 6 days progressively. After that, thrombocyte count slowly ascended to normal range within 15 days. She didn't take LMWH yet in this period. She was accepted as chemotherapy-responsive based on the examinations she has undergone after $6^{\text {th }}$ cycle of chemotherapy and therefore next planned $3^{\text {rd }}$ chemotherapy cycle of the interval debulking surgery was decided not to be given.

She was hospitalized after 47 days of the $6^{\text {th }}$ cycle of chemotherapy due to infection. Leukocytosis was present in hematologic parameters with neutrophil dominance where platelet count was $118.000 / \mathrm{mm}^{3}$. The origin of the infection was thought to be the lymphocyte in the abdomen thus IV antibiotic initiated. For prophylaxis due to hospitalization Clexane (4000 anti-Xa IU $40 \mathrm{mg}$ enoxaparin sodium) was administered. Pulmonary embolism was developed on the $2^{\text {nd }}$ day of inpatient follow-up so therefore heparin dose was increased to $2 \times 6000$ anti-Xa IU. After 5 days of LMWH use of treatment, a progressive decrease in platelets $(>50 \%)$ was observed. Considering all of the findings, 'HIT' was suspected and hereby heparin treatment was stopped. Fondaparinux treatment initiated and laboratory tests applied. Platelet levels returned to normal limits within 6 days. Clinical and biochemical infection findings were resolved on the $7^{\text {th }}$ day of IV antibiotics. The patient has taken fondaparinux treatment for more than 6 months.

Signed informed consent was obtained from the patient.

\section{Discussion}

Heparin-induced thrombocytopenia should be suspected in the patients who are currently receiving heparin or who received heparin and develops new onset of thrombocytopenia (i.e., platelet count $<150.000 / \mu \mathrm{L}$ ), a decrease in platelet count by 50 percent or more, even if the platelet count exceeds $150.000 / \mu \mathrm{L}$, venous or arterial thrombosis, necrotic skin lesions at heparin injection sites and acute systemic reactions (e.g., fever/chills, tachycardia, hypertension, dyspnea, cardiopulmonary arrest) occurring after intravenous heparin administration $(6,7)$.

Classically HIT related platelet decline is expected to occur within 5-10 days of heparin exposure but timing may show variability (8). Four types of clinical manifestations are defined in case of thrombocytopenia timing: 1. Typical-onset HIT, 2. Rapid-onset HIT, 3. Delayed-onset HIT and 4. Spontaneous HIT. Typical-onset HIT ( $70 \%$ of patients) manifests as a platelet count decrease that begins 5 to 10 days after starting a course of heparin. Rapid-onset HIT is the presenting feature in about one-quarter of patients and is defined as a decrease in platelet count within 24 hours of administering a course of heparin. Delayed-onset HIT refers to when the platelet count begins to decrease after all heparin has been stopped. Spontaneous HIT is a rare disorder in which a patient develops 
a clinical profile suggestive of HIT and in whom high levels of HIT antibodies can be detected, even though no heparin has been administered previously. The clinical progress of our case was mostly compatible with typical-onset HIT.

Factors such as; surgery, unfractionated heparin, female sex, older age increase the frequency of HIT. HIT occurs more commonly after exposure to UFH than exposure to LMWH in surgical patients (9). The pathophysiology of HIT may be different in unfractionated heparin than in LMWH because of the smaller molecule size of LMWH. (4) Ultra-large complexes form most efficiently with UFH; their formation is 10 -fold less efficient with LMWH and negligible with fondaparinux. These biochemical findings correlate with the greater incidence of HIT in patients receiving unfractionated compared with LMWH (1). This makes LMWH less likely to induce HIT than the larger unfractionated heparins (4). In a study of meta-analysis which aim was to determine the risk for HIT with unfractionated and low-molecular-weight heparin thromboprophylaxis, the absolute risk for HIT was only $0.2 \%$ with LMWH and was $2.6 \%$ with unfractionated heparin (9). Heparin dose is not relevant to the increasing risk of HIT. Therapeutic doses of heparin may result in a greater incidence of HIT than prophylactic doses, but there are no data that clearly define the relationship between heparin dose and clinical findings (10).

This case represents an example of HIT. In our case, progressive thrombocytopenia was observed after heparin treatment, pulmonary embolism was developed in consequence of thrombosis. Thus, HIT was suspected. The important point for clinicians about HIT is the decision to continue using heparin as a critical anticoagulant agent or cease the heparin therapy and choose an alternative non-heparin anticoagulant. The current treatment options for HIT are argatroban, fondaparinux, and bivalirudin. In the present case, fondaparinux was used.

A clinician should always consider clinical and laboratory evidence in evaluating patients for HIT. However, definitive laboratory data (i.e., immunoassay and/or functional assay for HIT antibodies) may not be available for several days. Since clinician needs to decide urgently, should make a preliminary diagnosis of HIT based on clinical findings and immediately available laboratory data. Clinicians can confirm or refute the diagnosis once they have the definitive results of HIT antibody testing. The 4Ts score may be used to have an early diagnose of HIT which the test includes; thrombocytopenia, the timing of the platelet decline, the presence of thrombosis, and other potential causes of thrombocytopenia (11) (Table I). The system yields an integer score between 0 and 8 with scores of 0 $3,4-5$, and 6-8 classified as low, intermediate, and high pretest probability for HIT, respectively (12). In a meta-analysis, the positive predictive value of high, intermediate, and combined high and intermediate probability 4Ts scores was $0.64,0.14$, and 0.22 respectively. The pooled estimates of sensitivity and specificity of the 4 Ts at a cut-off of $\geq 4$ were 0.99 and 0.54 , respectively. The 4Ts score was 8 in the presented case and accepted as high probability group.

In gynecologic oncology patients, there is no evidence data of HIT existence. However, in obstetrics patients which

Table I: 4Ts scoring system

\begin{tabular}{|c|c|c|c|}
\hline 4Ts scoring parameters & 2 Points & 1 Point & 0 Point \\
\hline $\begin{array}{l}\text { Thrombocytopenia score } \\
\text { Score of current case }=2\end{array}$ & $\begin{array}{l}>50 \% \text { platelet decrease to } \\
\text { nadir } \geq 20 \times 10^{9} / \mathrm{L}\end{array}$ & $\begin{array}{l}30 \%-50 \% \text { platelet count de- } \\
\text { crease or nadir } 10-19 \times 10 \% / \mathrm{L}\end{array}$ & $\begin{array}{l}<30 \% \text { platelet decrease or } \\
\text { nadir }<10 \times 10^{9} / \mathrm{L}\end{array}$ \\
\hline $\begin{array}{l}\text { Timing of platelet count de- } \\
\text { crease, thrombosis, or other } \\
\text { squeal (first day of heparin } \\
\text { course =day } 0 \text { ) } \\
\text { Score of current case=2 }\end{array}$ & $\begin{array}{l}\text { Day } 5-10 \text { onset or } \\
\leq 1 \text { day (with recent heparin ex- } \\
\text { posure within past } 5-30 \text { days) }\end{array}$ & $\begin{array}{l}\text { Consistent with day } 5-10 \\
\text { decrease, but not clear (e.g., } \\
\text { missing platelet counts), or } \leq 1 \\
\text { day (heparin exposure within } \\
\text { past } 31-100 \text { days), or platelet } \\
\text { decrease after day } 10\end{array}$ & $\begin{array}{l}\text { Platelet count decrease } \leq 4 \\
\text { days without recent heparin } \\
\text { exposure }\end{array}$ \\
\hline $\begin{array}{l}\text { Thrombosis (including adrenal } \\
\text { infarction) or other sequel } \\
\text { (e.g., skin lesions) } \\
\text { Score of current case=2 }\end{array}$ & $\begin{array}{l}\text { Proven new thrombosis, or } \\
\text { skin necrosis (at injection site), } \\
\text { or post-IV heparin bolus ana- } \\
\text { phylactic reaction }\end{array}$ & $\begin{array}{l}\text { Progressive or recurrent } \\
\text { thrombosis, or erythematous } \\
\text { skin lesions (at injection sites), } \\
\text { or suspected thrombosis (not } \\
\text { proven) }\end{array}$ & None \\
\hline $\begin{array}{l}\text { Other cause for } \\
\text { Thrombocytopenia } \\
\text { Score of current case=2 }\end{array}$ & $\begin{array}{l}\text { No explanation for platelet } \\
\text { count decrease is evident }\end{array}$ & Possible other cause is evident & Definite other cause is present \\
\hline Total score of current case & 8 & \multicolumn{2}{|c|}{ HIGH RISK } \\
\hline
\end{tabular}

The 4Ts score is the sum of the values for each of the 4 categories.

Scores of 1-3 considered to correspond to a low, Scores of 4-5 considered to correspond to an intermediate, Scores of 6-8 considered to correspond to a high probability 
UFH was used for prophylaxis, HIT was seemed to have a $1.5 \%$ rate (13). Greer and Nelson-Piercy, in their studies, defined that none of the 2777 patients who were used LMWH as prophylaxis, HIT was not detected (14).

Individuals with suspected HIT should stop using heparin immediately and therefore the patient should have a non-heparin treatment. Individuals with a confirmed diagnosis of HIT should continue the non-heparin anticoagulant approximately 3-6 months. The diagnosis of HIT is based on clinical features supported by laboratory testing which are immunoassays and functional assays. This case report describes a patient who was suspected of the diagnoses of HIT because of the clinical and laboratory findings and therefore heparin treatment was cancelled immediately and another anticoagulant treatment was initiated. Importantly, one should not wait for thrombosis to develop before suspecting HIT, because thrombocytopenia often precedes thrombosis. Early intervention has the potential to prevent thrombotic events, which are the major cause of morbidity and mortality in patients with HIT.

\section{Conclusion}

HIT is a temporary condition and the platelet count returns to normal within a few days to several weeks. HIT may both occur by the use of LMWH or UFH for the prophylaxis of thrombosis. By the use of fondaparinux, the platelet levels come to normal within 2-9 days. Misdiagnosis of HIT can have adverse outcomes. When the suspicion of HIT emerges, the clinician should use clinical probability scores and confirm the diagnosis with a peripheral blood sample and laboratory tests. HIT emerges rarely but the treatment is difficult so the treatment should be approached with care.

\section{Conflict of Interest Statement}

The authors declare no conflict of interest.

Author Contributions: YD: Concept, Data Collection, Data Processing, Writer. YAK: Concept, Data Collection, Data Processing, Writer. CK: Concept, Design, Writer. CC: Design, Writer. DY: Concept, Writer. BS: Concept, Writer. GB: Supervision, Concept, Design, Writer. GKC: Concept, Design, Writer. T T: Supervision, Concept, Design, Writer.

\section{References}

1. Linkins LA, Dans AL, Moores LK, Bona R, Davidson BL, Schulman S. et al. Treatment and prevention of heparininduced thrombocytopenia: Antithrombotic Therapy and Prevention of Thrombosis, 9th ed: American College of Chest Physicians Evidence-Based Clinical Practice Guidelines. Chest. 2012;141(2, Suppl): e495S-e530S.
2. Keeling D, Davidson S, Watson H. The management of heparin-induced thrombocytopenia. Br J Haematol. 2006; 133(3):259-69.

3. Salter BS, Weiner MM, Trinh MA, Heller J, Evans AS, Adams DH. et al. Heparin-induced thrombocytopenia: a comprehensive clinical review. J Am Coll Cardiol. 2016;67(21):2519-32.

4. Greinacher A, Völpel H, Janssens U, Hach-Wunderle V, Kemkes-Matthes B, Eichler P, et al. Recombinant hirudin (Lepirudin) provides safe and effective anticoagulation in patients with heparin induced thrombocytopenia. Circulation. 1999;99(1):73-80.

5. LaMonte M P, Brown P M, Hursting M J. Stroke in patients with heparin-induced thrombocytopenia and the effect of argatroban therapy. Crit Care Med. 2004;32(4): 976-80.

6. Warkentin TE, Greinacher A, Koster A, Lincoff AM. Treatment and prevention of heparin-induced thrombocytopenia: American College of Chest Physicians EvidenceBased Clinical Practice Guidelines ( $8^{\text {th }}$ Edition). 2008;133(6 Suppl):340S.

7. Warkentin TE. Thromb Haemost. Clinical picture of heparin-induced thrombocytopenia (HIT) and its differentiation from non-HIT thrombocytopenia. Thromb Haemost. 2016;116(5):813-22.

8. Favaloro EJ, McCaughan G, Pasalic L. Clinical and laboratory diagnosis of heparin induced thrombocytopenia: an update. Pathology. 2017;49(4):346-55.

9. Martel N, Lee J, Wells PS. Risk for heparin-induced thrombocytopenia with unfractionated and low-molecular-weight heparin thromboprophylaxis: a meta-analysis. Blood. 2005;106(8):2710-5.

10. Smythe MA, Koerber JM, Mattson JC. The incidence of recognized heparin-induced thrombocytopenia in a large, tertiary care teaching hospital. 2007;131(6):1644-9.

11. www.hematology.org/Clinicians/Guidelines-Quality/ Guidelines.aspx. (2018).

12. Lo GK, Juhl D, Warkentin TE, Sigouin CS, Eichler P, Greinacher A. Evaluation of pretest clinical score (4 T's) for the diagnosis of heparin-induced thrombocytopenia in two clinical settings. J Thromb Haemost. 2006; 4(4):759-6.

13. Sagaram D, Siddiq Z, Eisenberger AB, Ananth CV, Wright JD, D'Alton ME, et al. Heparin-Induced thrombocytopenia during obstetric hospital admissions. Am J Perinatol. 2018;35(9):898-903.

14. Greer IA, Nelson-Piercy C. Low-molecular-weight heparins for thromboprophylaxis and treatment of venous thromboembolism in pregnancy: a systematic review of safety and efficacy. Blood. 2005;106(02):401-7. 